\title{
Probing the equation of state of neutron-rich matter with intermediate energy heavy-ion collisions
}

BaO-An Li ${ }^{1}$, Lie-Wen Chen ${ }^{2}$, Che Ming $\mathrm{Ko}^{3}$ and Andrew W. Steiner ${ }^{4}$

\author{
${ }^{1}$ Department of Chemistry and Physics, P.O. Box 419, \\ Arkansas State University, State University, AR 72467-0419, USA \\ ${ }^{2}$ Institute of Theoretical Physics, Shanghai Jiao Tong University, \\ Shanghai 200240, and Center of Theoretical Nuclear Physics, National \\ Laboratory of Heavy-Ion Accelerator, Lanzhou, 730000, China \\ ${ }^{3}$ Cyclotron Institute and Physics Department, Texas A\&M University, \\ College Station, Texas 77843, USA \\ 4 Theoretical Division, Los Alamos National Laboratory, \\ Los Alamos, NM 87545, USA
}

\begin{abstract}
Nuclear reactions induced by stable and/or radioactive neutronrich nuclei provide the opportunity to pin down the equation of state of neutron-rich matter, especially the density $(\rho)$ dependence of its isospin-dependent part, i.e., the nuclear symmetry energy $E_{\text {sym. }}$ A conservative constraint, $32\left(\rho / \rho_{0}\right)^{0.7}<E_{\text {sym }}(\rho)<32\left(\rho / \rho_{0}\right)^{1.1}$, around the nuclear matter saturation density $\rho_{0}$ has recently been obtained from the isospin diffusion data in intermediate energy heavy-ion collisions. We review this exciting result and discuss its consequences and implications on nuclear effective interactions, radii and cooling mechanisms of neutron stars.
\end{abstract}



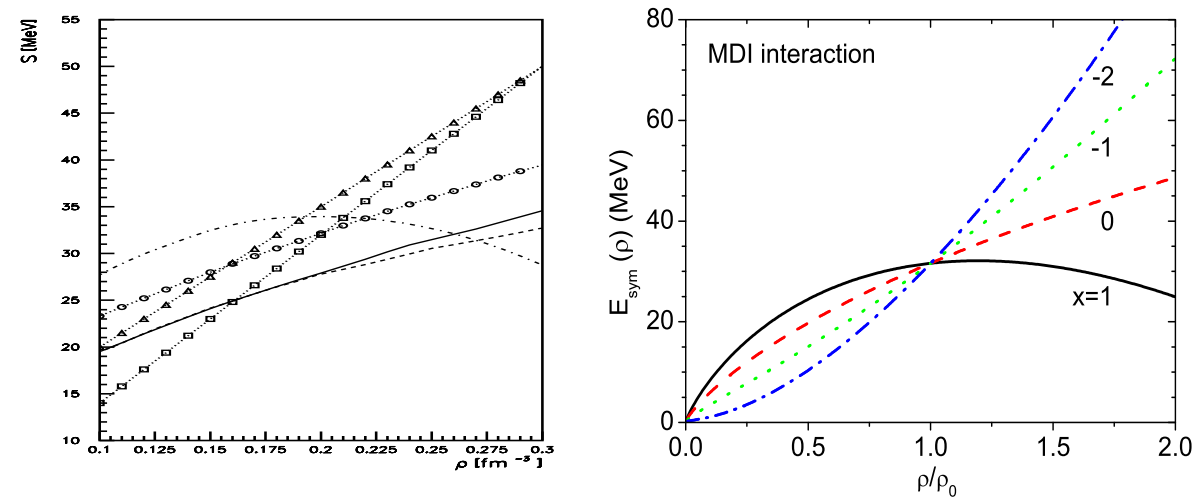

Figure 1: Left panel: density dependence of the symmetry energy $S$ from the continuous choice Brueckner-Hartree-Fock with Reid93 potential (circles), self-consistent Green's function theory with Reid93 potential (full line), variational calculation with Argonne Av14 potential (dashed line), Dirac-Brueckner-Hartree-Fock calculation (triangles), relativistic mean-field model (squares), and effective field theory (dash-dotted line) 9 . Right panel: same as left panel for the MDI interaction with four $x$ parameters as used in the IBUU04 model 10.

\section{Nuclear equation of state and symmetry energy of neutron-rich matter}

Using a parabolic approximation which has been verified by all many-body theories to date, the equation of state (EOS) of isospin asymmetric nuclear matter can be written as

$$
E(\rho, \delta)=E(\rho, \delta=0)+E_{\mathrm{sym}}(\rho) \delta^{2}+\mathcal{O}\left(\delta^{4}\right),
$$

where $\delta \equiv\left(\rho_{n}-\rho_{p}\right) /\left(\rho_{p}+\rho_{n}\right)$ is the isospin asymmetry and $E_{\text {sym }}(\rho)$ is the density-dependent nuclear symmetry energy. The latter is very important for understanding many interesting astrophysical problems [1, 2], the structure of radioactive nuclei 3, 4, and the dynamics of heavy-ion reactions [5, 6, 7, 8]. Unfortunately, $E_{\mathrm{sym}}(\rho)$ is poorly known with theoretical predictions diverging widely at both low and high densities as shown in the left panel of Fig. 1 1 for some of the most widely used microscopic many-body theories 9. The theoretical uncertainties are largely due to a lack of knowledge about the isospin dependence of nuclear effective interactions and the limitations of existing many-body techniques. On the other hand, heavy-ion reactions, especially those induced by radioactive beams, provide a unique opportunity to pin down the density dependence of nuclear symmetry en- 
ergy in terrestrial laboratories. Indeed, significant progress has recently been made both experimentally and theoretically in determining the symmetry energy at subnormal densities [10, 11, 12, 13, 14]. Future high energy radioactive beams will further allow one to determine the symmetry energy at supranormal densities.

To extract information about the density dependence of the nuclear symmetry energy from nuclear reactions induced by neutron-rich nuclei, reliable reaction models that take into account the isospin degree of freedom are needed. One such model that has been used extensively is the isospinand momentum-dependent IBUU04 transport model [15]. In this model, the single-nucleon potential, which is one of the most important inputs to all transport models, has the following density- and momentum-dependent form [16]

$$
\begin{aligned}
& U(\rho, \delta, \mathbf{p}, \tau, x)=A_{u}(x) \frac{\rho_{\tau^{\prime}}}{\rho_{0}}+A_{l}(x) \frac{\rho_{\tau}}{\rho_{0}}+B\left(\frac{\rho}{\rho_{0}}\right)^{\sigma}\left(1-x \delta^{2}\right) \\
& -8 \tau x \frac{B}{\sigma+1} \frac{\rho^{\sigma-1}}{\rho_{0}^{\sigma}} \delta \rho_{\tau^{\prime}}+\sum_{t=\tau, \tau^{\prime}} \frac{2 C_{\tau, t}}{\rho_{0}} \int d^{3} \mathbf{p}^{\prime} \frac{f_{t}\left(\mathbf{r}, \mathbf{p}^{\prime}\right)}{1+\left(\mathbf{p}-\mathbf{p}^{\prime}\right)^{2} / \Lambda^{2}}
\end{aligned}
$$

where $x, A_{u}(x), A_{\ell}(x), B, C_{\tau, \tau^{\prime}}, \sigma$, and $\Lambda$ are all parameters. The corresponding momentum-dependent interaction (MDI) gives an incompressibility of $K_{0}=211 \mathrm{MeV}$ for symmetric nuclear matter at saturation density. The parameter $x$ is introduced to mimic predictions on $E_{\text {sym }}(\rho)$ by microscopic and/or phenomenological many-body theories [15. Shown in the right panel of Fig. 1 is the density dependence of the symmetry energy for $x=-2,-1,0$ and 1 . The momentum dependence of the single-nucleon potential, given by the last term of Eq. (2), leads to reduced effective masses for nucleons and consequently also the in-medium nucleon-nucleon (NN)

cross sections [13, 17. In the IBUU04 transport model, both experimental free-space and in-medium NN cross sections can be used.

\subsection{Constraining the density dependence of the nuclear sym- metry energy by isospin diffusion}

Tsang et al. 11 recently studied the degree of isospin diffusion in the reaction ${ }^{124} \mathrm{Sn}+{ }^{112} \mathrm{Sn}$ by measuring [18]

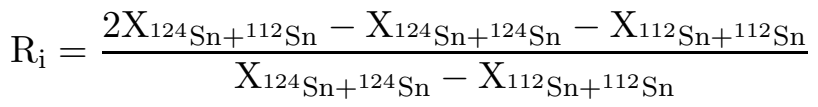

where $\mathrm{X}$ is the average isospin asymmetry $\langle\delta\rangle$ of the ${ }^{124}$ Sn-like residue. Shown in the left panel of Fig. 2 is a comparison of the strength of isospin 

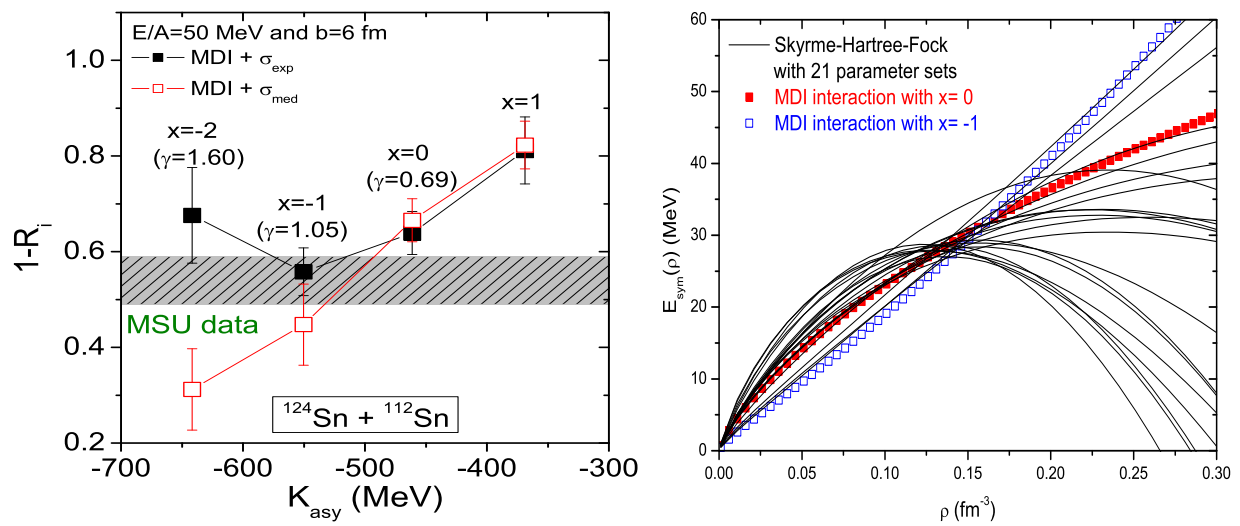

Figure 2: Left panel: the degree of isospin diffusion as a function of $K_{\text {asy }}\left(\rho_{0}\right)$ for free (filled squares) and in-medium (open squares) nucleon-nucleon cross sections [13]. Right panel: symmetry energies obtained from 21 sets of Skyrme interactions and the MDI interaction with $x=-1$ and $x=0$ [14].

diffusion $1-R_{i}$ obtained with free (filled squares) and in-medium (open squares) NN cross sections as a function of the asymmetric part of the isobaric incompressibility of nuclear matter at saturation density $\rho_{0}$ [10], i.e.,

$$
K_{\text {asy }}\left(\rho_{0}\right) \equiv 9 \rho_{0}^{2}\left(d^{2} E_{\text {sym }} / d \rho^{2}\right)_{\rho_{0}}-18 \rho_{0}\left(d E_{\text {sym }} / d \rho\right)_{\rho_{0}} .
$$

It is interesting to note that with in-medium $\mathrm{NN}$ cross sections the strength of isospin diffusion $1-\mathrm{R}_{\mathrm{i}}$ decreases monotonically with decreasing value of $x$ (i.e., increasing stiffness). With free-space NN cross sections, there appears, however, a minimum at around $x=-1$. The value of $K_{\text {asy }}\left(\rho_{0}\right)$ determined from the use of in-medium NN cross sections in the IBUU04 model is about $-500 \pm 50 \mathrm{MeV}$. This value is more precise than previous estimates [19] and thus represents a significant advance in resolving the major remaining uncertainty about the incompressibility of nuclear matter at normal density [20, 21]. Also shown in Fig. 2 are the values for $\gamma$ used in fitting the symmetry energy around $\rho_{0}$ using the expression $E_{\text {sym }}(\rho)=$ $32\left(\rho / \rho_{0}\right)^{\gamma}$. From results obtained with the in-medium NN cross sections, the $\gamma$ parameter is constrained to be between 0.7 and 1.1.

\section{Constraining nuclear effective interactions}

The above constraints on the symmetry energy limit the nuclear effective interactions in nuclear matter. This can be seen by comparing them with 
the symmetry energies obtained from Skyrme effective interactions 14. The right panel of Fig. 2 displays the density dependence of $E_{\text {sym }}(\rho)$ for 21 sets of Skyrme interaction parameters. Most of these effective interactions are not compatible with that extracted from the isospin diffusion data, given by the MDI interaction with $x$ parameter between -1 and 0 , except the SIV, $\mathrm{SV}, \mathrm{G}_{\sigma}$, and $\mathrm{G}_{R}$ Skyrme effective interactions.

\section{Constraining the slope of the symmetry energy}
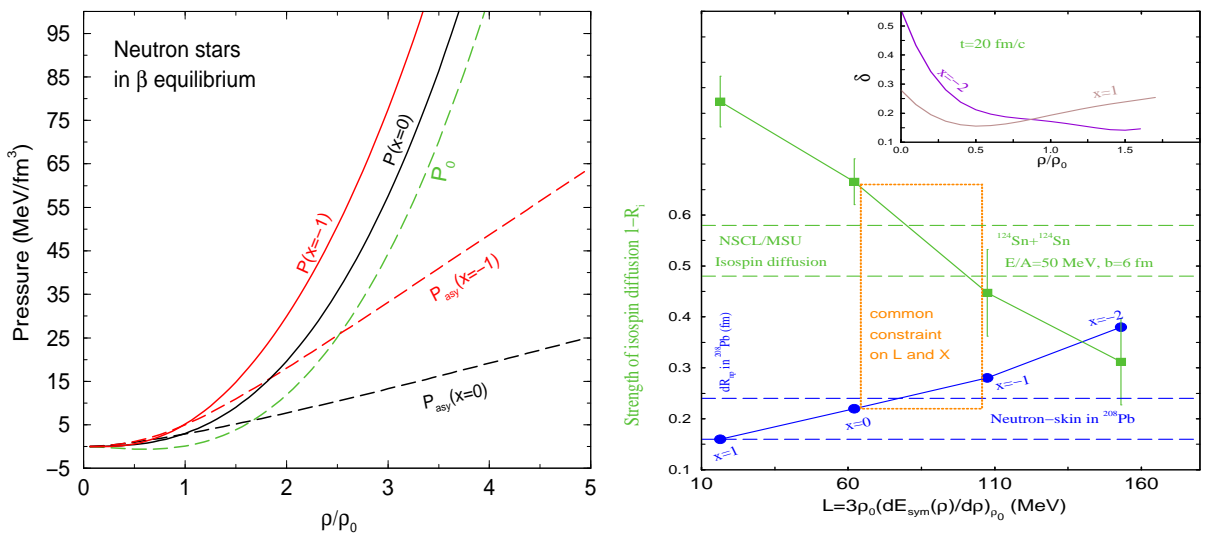

Figure 3: Left panel: symmetric $\left(P_{0}\right)$, asymmetric $\left(P_{\text {asy }}\right)$, and total pressure in neutron stars at $\beta$-equilibrium using the MDI interaction with $x=0$ and $x=-1$. Right panel: strength of isospin diffusion in ${ }^{124} \mathrm{Sn}+{ }^{112} \mathrm{Sn}$ reactions and size of neutron skin in ${ }^{208} \mathrm{~Pb}$ as functions of the slope of the symmetry energy. The inset is the correlation between isospin asymmetry and density of matters at the instant of $20 \mathrm{fm} / \mathrm{c}$ in the considered reaction [22].

While most properties of neutron stars depend on both the isospinindependent and -dependent parts of the nuclear equation of state, their radii are primarily determined by the isospin asymmetric pressure that is proportional to the slope of the symmetry energy $E_{\mathrm{sym}}^{\prime}(\rho)[1]$. For the simplest case of a neutron-proton-electron (npe) matter in neutron stars at $\beta$ equilibrium, the pressure is given by

$$
\begin{aligned}
P(\rho, \delta) & =P_{0}(\rho)+P_{\text {asy }}(\rho, \delta)=\rho^{2}\left(\frac{\partial E}{\partial \rho}\right)_{\delta}+\frac{1}{4} \rho_{e} \mu_{e} \\
& =\rho^{2}\left[E^{\prime}(\rho, \delta=0)+E_{\mathrm{sym}}^{\prime}(\rho) \delta^{2}\right]+\frac{1}{2} \delta(1-\delta) \rho E_{\mathrm{sym}}(\rho)
\end{aligned}
$$

where the electron density is $\rho_{e}=\frac{1}{2}(1-\delta) \rho$ and the chemical potential is 
$\mu_{e}=\mu_{n}-\mu_{p}=4 \delta E_{\mathrm{sym}}(\rho)$. The equilibrium value of $\delta$ is determined by the chemical equilibrium and charge neutrality conditions, i.e., $\delta=1-2 x_{p}$ with

$$
x_{p} \approx 0.048\left[E_{\mathrm{sym}}(\rho) / E_{\mathrm{sym}}\left(\rho_{0}\right)\right]^{3}\left(\rho / \rho_{0}\right)\left(1-2 x_{p}\right)^{3} .
$$

Because of the large $\delta$ value in neutron stars, the electron degenerate pressure is small. Moreover, the isospin symmetric contribution to the pressure is also very small around normal nuclear matter density as $E^{\prime}\left(\rho_{0}, \delta=0\right)=0$. Shown in the left panel of Fig. 3 are the isospin symmetric $\left(P_{0}\right)$ and asymmetric $\left(P_{a s y}\right)$ as well as the total pressure in neutron stars at $\beta$-equilibrium calculated from Eq.(5D) using the MDI interaction with $x=0$ and $x=-1$. It is seen that up to about $2.5 \rho_{0}$ for $x=-1$ and about $1.5 \rho_{0}$ for $x=0$ the total pressure is dominated by the isospin asymmetric contribution. Because neutron star radii are determined by the pressure at moderate densities where the proton content of matter is small, they are very sensitive to the slope of the symmetry energy near and just above $\rho_{0}$. In particular, a stiffer symmetry energy is expected to lead to a larger neutron star radius.

For isospin diffusion in heavy-ion reactions, it is a re-distribution of isospin asymmetries that is initially carried by the colliding nuclei. The degree and rate of this process depend on the relative pressures of neutrons and protons, namely the slope of $E_{\mathrm{sym}}(\rho)$. With a stiffer $E_{\mathrm{sym}}(\rho)$, it is harder for neutrons and protons to mix, leading thus to a smaller/slower isospin diffusion. Because of isospin asymmetric pressure, dilute neutron-rich clouds surrounding a more symmetric dense region are dynamically generated in heavy-ion reactions through isospin diffusion as illustrated in the inset of the right panel of Fig. 3, where the correlation between isospin asymmetry and density of matters at the instant of $20 \mathrm{fm} / \mathrm{c}$ in ${ }^{124} \mathrm{Sn}+{ }^{112} \mathrm{Sn}$ reactions is shown. A similar effect is seen in neutron-rich nuclei, where the isospin asymmetric pressure pushes extra neutrons further out of an isospin symmetric core of near normal density, leading to an increasing of the sizes of neutron-skins in neutron-rich nuclei with increasing slope of the symmetry energy [2, 3, 23.

From above discussions, one expects that the radii of neutron stars, the degree of isospin diffusion in heavy-ion collisions, and the sizes of neutronskins in heavy nuclei are all correlated through the same underlying nuclear symmetry energy. This is demonstrated in the right panel of Fig. 3, where the strength of the isospin diffusion $1-R_{i}$, calculated with the IBUU04 model with in-medium NN cross sections, and the thickness of neutron skin $d R_{n p}$ in ${ }^{208} \mathrm{~Pb}$, calculated using the Skyrme Hartree-Fock with interaction parameters adjusted to give an EOS which is similar to the effective interaction used in the IBUU04 model [12, are examined simultaneously as 
functions of the slope parameter $L \equiv 3 \rho_{0}\left(\partial E_{\text {sym }} / \partial \rho\right)_{\rho_{0}}$ of the symmetry energy. It is seen that $1-R_{\mathrm{i}}$ decreases while $d R_{n p}$ increases with increasing $L$ as expected. Taken the fiducial value $d R_{n p}=0.2 \pm 0.04 \mathrm{fm}$, that is measured and supported strongly by several recent calculations [2], and the $\mathrm{NSCL} / \mathrm{MSU}$ data $1-\mathrm{R}_{\mathrm{i}}=0.525 \pm 0.05$ [1], the $L$ parameter is constrained in a common range between $62.1 \mathrm{MeV}(x=0)$ and $107.4 \mathrm{MeV}(x=-1)$ [22].

\section{Constraining the radii and cooling mechanisms of neutron stars}
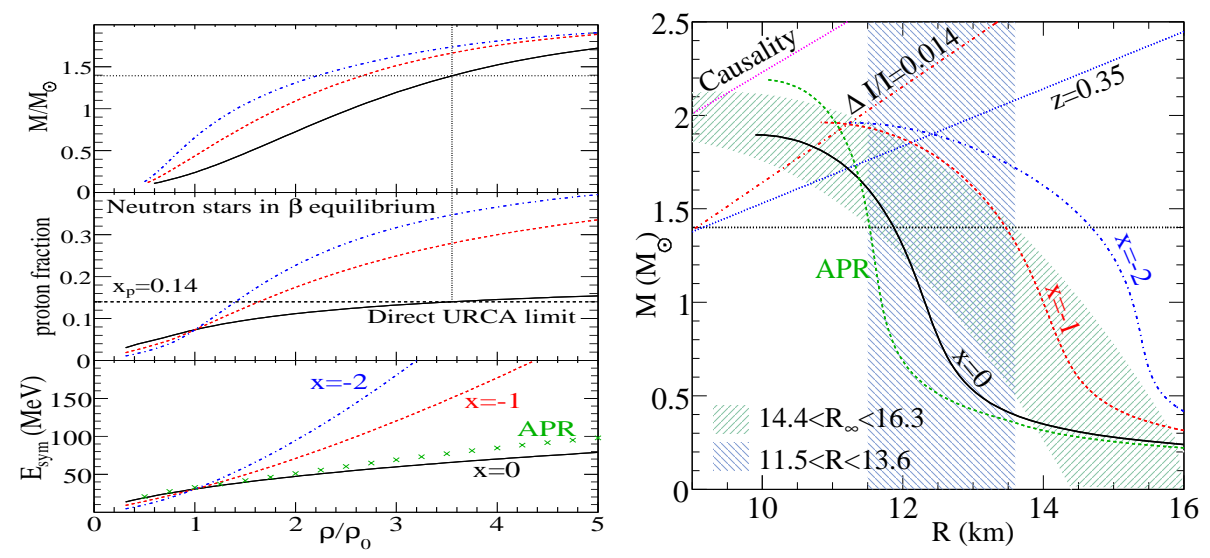

Figure 4: Left panel: mass and proton fraction of neutron star and the symmetry energy as functions of density. Right panel: correlations in neutron star masses and radii 22. All results are for spherically-symmetric, non-rotating, non-magnetized neutron stars consisting of npe $\mu$ matter at zero temperature.

The dependence of some of the basic properties of neutron stars on the nuclear EOS is shown in the left panel of Fig. 4. The top and middle panels give, respectively, the mass of neutron star and its proton fraction $x_{p}$, calculated from the MDI interaction with $x=0,-1$, and -2 , as functions of its central density. The symmetry energies obtained from these interactions are shown in the lower panel together with that from the AV18+ $\delta v+\mathrm{UIX}^{*}$ interaction of Akmal et al. (APR) 24]. It is interesting to see that up to about $5 \rho_{0}$ the symmetry energy predicted by APR agrees very well with that from the MDI interaction with $x=0$ and is thus within the range of symmetry energies constrained by both isospin diffusion and ${ }^{208} \mathrm{~Pb}$ neutronskin thickness data. Because of the lack of experimental information on the 
EOS of pure neutron matter, the APR prediction has been widely used as a standard in calibrating other microscopic calculations.

For $x_{p}$ below 0.14 [1] , the direct URCA process for fast cooling of protoneutron stars does not proceed because energy and momentum conservation cannot be simultaneously satisfied. For EOSs from the MDI interaction with $x=-1$ and $x=-2$, the condition for direct URCA process is fulfilled for nearly all neutron stars above $1 \mathrm{M}_{\odot}$. For the EOS from $x=0$, the minimum density for the direct URCA process is indicated by the vertical dotted line, and the corresponding minimum neutron star mass is indicated by the horizontal dotted line. Neutron stars with masses above $1.39 \mathrm{M}_{\odot}$ are thus expected to have a central density above the threshold for the direct URCA process.

The relations between neutron star masses and radii for above EOSs are given in the right panel of Fig. 4. Also given are the constraints due to causality as well as the mass-radius relations from estimates of the crustal fraction of the moment of inertia $(\Delta I / I=0.014)$ in the Vela pulsar [25] and from the redshift measurement from Ref. [26]. Allowed equations of state should lie to the right of the causality line and also cross the other two lines. The hatched regions are inferred limits on the radius and the radiation radius (the value of the radius which observed by an observer at infinity) defined as $R_{\infty}=R / \sqrt{1-2 G M / R c^{2}}$ for a $1.4 \mathrm{M}_{\odot}$ neutron star. It is seen that the symmetry energy affects strongly the radius of a neutron star but only slightly its maximum mass [1, 27. These analyses have led to the conclusion that only radii between 11.5 and $13.6 \mathrm{~km}$ (or radiation radii between 14.4 and $16.3 \mathrm{~km}$ ) are consistent with the EOSs from the MDI interaction with $x=0$ and $x=-1$ and thus with the laboratory data on isospin diffusion and neutron skin thickness of heavy nuclei [22].

The observational determination of the neutron star radius from the measured spectral fluxes relies on a numerical model of the neutron star atmosphere and uses as inputs the composition of the atmosphere, a measurement of the distance, the column density of x-ray absorbing material, and the surface gravitational redshift. Since many of these quantities are difficult to measure, only a paucity of radius measurements are available. Current estimates obtained from recent $\mathrm{x}$-ray observations give a wide range of results. It is therefore very useful to compare our results with recent Chandra/XMM-Newton observations. Assuming a mass of $1.4 \mathrm{M}_{\odot}$, the inferred radiation radius $R_{\infty}$ (in $\mathrm{km}$ ) is $13.5 \pm 2.1$ [28] or $13.6 \pm 0.3$ [29] for the neutron star in $\omega$ Cen, $12.8 \pm 0.4$ in M13 [30], $14.5_{-1.4}^{+1.6}$ for X7 in 47 Tuc [31, and $14.5_{-3.8}^{+6.9}$ in M28 32 . Except the neutron star in M13 that has a slightly smaller radius, all others fall into our constraints of $14.4 \mathrm{~km}<R_{\infty}<16.3$ 
$\mathrm{km}$ within the observational error bars that are larger than the above range in most cases.

\section{Summary}

The EOS of neutron-rich matter is fundamentally important for both nuclear physics and astrophysics. Nuclear reactions induced by neutron-rich nuclei provide a great opportunity to constrain the EOS of neutron-rich matter. The recent analysis of isospin diffusion data in heavy-ion collisions within the IBUU04 transport model allowed us to constrain the density dependence of the nuclear symmetry energy in a narrow range. Together with experimental information on the neutron-skin thickness of ${ }^{208} \mathrm{~Pb}$, this has led to stringent constraints on not only the nuclear effective interactions but also the cooling mechanisms and radii of neutron stars.

This Work work was supported in part by the US National Science Foundation under Grant No. PHYS-0354572 and PHYS-0456890, and the NASAArkansas Space Grants Consortium award ASU15154 (BAL); National Natural Science Foundation of China under Grant No. 10105008 and 10575071 (LWC); the US National Science Foundation under Grant No. PHY-0457265 and the Welch Foundation under Grant No. A-1358 (CMK); and the US Department of Energy under grant No. DOE/W-7405-ENG-36 (AWS).

\section{References}

[1] J.M. Lattimer and M. Prakash, Phys. Rep., 333, 121 (2000); Astrophys. J. 550, 426 (2001); Science 304, 536 (2004).

[2] A. W. Steiner, M. Prakash, J.M. Lattimer, and P.J. Ellis, Phys. Rep. 411, $325(2005)$.

[3] B.A. Brown, Phys. Rev. Lett. 85, 5296 (2000).

[4] J.R. Stone et al., Phys. Rev. C68, 034324 (2004).

[5] B.A. Li, C.M. Ko, and W. Bauer, Int. J. Mod. Phys. E7, 147 (1998).

[6] Isospin Physics in Heavy-Ion Collisions at Intermediate Energies, Eds. B. A. Li and W. Udo Schröder (Nova Science Publishers, Inc, New York, 2001).

[7] P. Danielewicz, R. Lacey, and W.G. Lynch, Science 298, 1592 (2002). 
[8] V. Baran, M. Colonna, V. Greco, and M. Di Toro, Phys. Rep. 410, 335 (2005).

[9] A.E.L. Dieperink et al., Phys. Rev. C68, 064307 (2003).

[10] L.W. Chen, C.M. Ko, and B.A. Li, Phys. Rev. Lett. 94, 032701 (2005).

[11] M.B. Tsang et al., Phys. Rev. Lett. 92, 062701 (2004).

[12] A.W. Steiner and B.A. Li, Phys. Rev. C72, 041601 (R) (2005).

[13] B.A. Li and L.W. Chen, Phys. Rev. C72, 064611 (2005).

[14] L.W. Chen, C.M. Ko, and B.A. Li, Phys. Rev. C72, 064309 (2005).

[15] B.A. Li, C.B. Das, S. Das Gupta, and C. Gale, Phys. Rev. C69, 011603 (2004); Nucl. Phys. A735, 563 (2004).

[16] C.B. Das, S. Das Gupta, C. Gale, and B.A. Li, Phys. Rev. C67, 034611 (2003).

[17] F. Sammrruca and P. Krastev, nucl-th/0506081, Phys. Rev. C in press.

[18] F. Rami et al., Phys. Rev. Lett. 84, 1120 (2000).

[19] S. Shlomo and D. H. Youngblood, Phys. Rev. C 47, 529 (1993).

[20] J. Piekarewicz, Phys. Rev. C 69, 041301 (2004).

[21] G. Colo et al., Phys. Rev. C 70, 024307 (2004).

[22] B.A. Li and A.W. Steiner, nucl-th/0511064.

[23] C.J. Horowitz and J. Piekarewicz, Phys. Rev. Lett. 86, 5647 (2001).

[24] A. Akmal, V.R. Pandharipande, and D.G. Ravenhall, Phys. Rev. C58, 1804 (1998).

[25] B. Link, R. I. Epstein, and J.M. Lattimer; Phys. Rev. Lett. 83, 3362 (1999).

[26] J. Cottam, F. Paerels, and M. Mendez, Nature 420, 51 (2002).

[27] M. Prakash et al., Phys. Rev. Lett. 61, 2518 (1988).

[28] R.E. Rutledge et al., Astrophys. J. 580, 413 (2002); ibid, 577, 346 (2002).

[29] B. Gendre, D. Barret, and N. A. Webb, Astron. Astrophys. 400, 521 (2003).

[30] B. Gendre, D. Barret, and N. Webb, Astron. Astrophys. 403, L11 (2003).

[31] G.B. Rybicki et al., astro-ph/0506563.

[32] W. Becker et al., Astrophys. J. 594, 798 (2003). 